\title{
NUMERICAL MODELLING OF THE ENDOTHERMIC PROCESS OF THE STEAM REFORMING OF METHANE IN A FIXED BED REFORMER
}

\author{
D. R. Dessaune, \\ V. F. Dias, \\ J. D. Silva, \\ Polytechnic School - UPE, Laboratory of \\ Environmental and Energetic Technology; Rua \\ Benfica - 455, Madalena, Recife - PE, Brazil, \\ Cep: $50750-470$ \\ daniel.dessaune@gmail.com \\ vi.dias11@hotmail.com \\ jornandesdias@poli.br \\ ABSTRACT \\ Thermochemical Packed-Bed (TPB) reformer has been substantially studied \\ in the past years as a promising equipment to investigate the \\ thermochemical conversion of methane $\left(\mathrm{CH}_{4}\right)$. This work has as main \\ objective a theoretical modelling to describe the process variables of Steam \\ Reforming of Methane (SRM) method in the TPB reformer. The TPB \\ reformer is filled with $\beta$-SiC open-cell foam where the thermochemical \\ conversion of $\mathrm{CH}_{4}$ is carried out. The model variables describe the specific \\ aims of work and these objectives can be identified from each equation of \\ the developed mathematical model. This work has been proposed to study \\ two specific aims as (i) the effective thermal conductivity's effect of the \\ solid phase $\left(\lambda_{\mathrm{s}, \text { eff. }}\right)$ and (ii) molar flows of chemical components. The \\ endothermic reaction temperature's profiles are notably increased as the

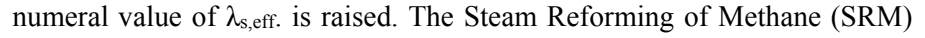 \\ method is suggested to improve the Production Rate (PR) of $\mathrm{H}_{2}$ regarding \\ the PR of CO. As results, the PR of $\mathrm{H}_{2}$ is of $29.48 \%$ while the PR of $\mathrm{CO}$ is \\ Received: Dec 01, 2020 \\ of $2.76 \%$. \\ Revised: Jan 04, 2021 \\ Accepted: Jan 08, 2021 \\ Keywords: $\beta$-SiC open-cell foam, TPB reformer, reaction temperature, \\ SRM method, mathematical model
}

\begin{tabular}{|c|c|}
\hline \multicolumn{2}{|c|}{ NOMENCLATURE } \\
\hline $\mathrm{C}_{\mathrm{p}, \mathrm{g}, \text { mix }}$ & $\begin{array}{l}\text { Molar heat capacity at constant pressure of } \\
\text { the gas mixture, } \mathrm{kJ} / \mathrm{kg} \mathrm{K}\end{array}$ \\
\hline $\mathrm{C}_{\mathrm{p}, \mathrm{s}}$ & $\begin{array}{l}\text { Heat capacity pressure of the solid phase, } \\
\mathrm{kJ} / \mathrm{kg} \mathrm{K}\end{array}$ \\
\hline $\mathrm{D}_{\mathrm{ax}, \mathrm{i}}$ & $\begin{array}{l}\text { Dispersion coefficients of each component } \mathrm{i} \text {, } \\
\mathrm{m}^{2} / \mathrm{s}\end{array}$ \\
\hline$d_{p}$ & Diameter of the solid particles, $\mathrm{m}$ \\
\hline $\mathrm{F}_{\mathrm{i}}$ & Molar flow of components i, kmol/s \\
\hline $\mathrm{F}_{\mathrm{j}, 0}$ & Initial molar flows of components $\mathrm{j}, \mathrm{kmol} / \mathrm{s}$ \\
\hline $\mathrm{g}$ & Gravity acceleration, $\mathrm{m} / \mathrm{s}^{2}$ \\
\hline $\mathrm{h}_{\mathrm{gs}}$ & Gas-solid heat transfer coefficient, $\mathrm{W} / \mathrm{m}^{2} \mathrm{~K}$ \\
\hline $\mathrm{k}_{\mathrm{SRM}}$ & $\begin{array}{l}\text { Reaction constant of the } \mathrm{R}_{\mathrm{SRM}}, \mathrm{kmol} \\
\mathrm{kPa}^{0.5} / \mathrm{kg}_{\text {cat. }} \mathrm{S}\end{array}$ \\
\hline $\mathrm{k}_{\mathrm{WGSR}}$ & $\begin{array}{l}\text { Reaction constant of the } \mathrm{R}_{\text {WGSR }}, \mathrm{kmol} \mathrm{kPa}^{-1} / \\
\mathrm{kg}_{\text {cat. }} \mathrm{S}\end{array}$ \\
\hline $\mathrm{K}_{\mathrm{SRM}}$ & Equilibrium constant of the $\mathrm{R}_{\mathrm{SRM}}, \mathrm{kPa}^{2}$ \\
\hline $\mathrm{K}_{\mathrm{WGSR}}$ & Equilibrium constant of the $\mathrm{R}_{\mathrm{WGSR}}$ \\
\hline $\mathrm{K}_{\mathrm{i}}$ & $\begin{array}{l}\text { Adsorption constants of components } \mathrm{i}, \mathrm{i}= \\
\mathrm{CO}, \mathrm{H}_{2} \text { and } \mathrm{CH}_{4}, \mathrm{kPa}^{-1}\end{array}$ \\
\hline $\mathrm{K}_{\mathrm{j}}$ & $\begin{array}{l}\text { Adsorption constant of components } \mathrm{j}, \mathrm{j}= \\
\mathrm{H}_{2} \mathrm{O}\end{array}$ \\
\hline $\mathrm{L}$ & TPB reformer length, $\mathrm{m}$ \\
\hline $\mathrm{P}_{\mathrm{i}}$ & $\begin{array}{l}\text { Partial pressures of components } \mathrm{i}, \mathrm{i}=\mathrm{CH}_{4} \text {, } \\
\mathrm{H}_{2} \mathrm{O}, \mathrm{CO}, \mathrm{CO}_{2} \text { and } \mathrm{H}_{2}, \mathrm{kPa}\end{array}$ \\
\hline Pop. & Operating pressure, $\mathrm{kPa}$ \\
\hline $\mathrm{q}_{\mathrm{g}}$ & Gas phase's flow rate, $\mathrm{m}^{3} / \mathrm{s}$ \\
\hline$r_{i}$ & $\begin{array}{l}\text { Net rates of components i }\left(\mathrm{i}=\mathrm{CH}_{4}, \mathrm{H}_{2} \mathrm{O} \text {, }\right. \\
\left.\mathrm{CO}, \mathrm{CO}_{2} \text { and } \mathrm{H}_{2}\right), \mathrm{kmol} / \mathrm{kg}_{\text {cat. }} \mathrm{s}\end{array}$ \\
\hline $\mathrm{R}_{\mathrm{i}}$ & $\begin{array}{l}\text { Overall rates of the reforming reactions } \mathrm{j}, \mathrm{j}= \\
1 \text { and } 2, \mathrm{kmol} / \mathrm{kg}_{\text {cat. }} \mathrm{s}\end{array}$ \\
\hline
\end{tabular}
$\mathrm{S}_{\mathrm{sp}} \quad$ Spatial velocity, $\mathrm{s}^{-1}$
$\mathrm{t} \quad$ Iteration time, $\mathrm{s}$
$\mathrm{T}_{\mathrm{er}, 0} \quad$ Initial temperature of the endothermic reaction, $\mathrm{K}$
$\mathrm{T}_{\mathrm{er}} \quad$ Temperature of the endothermic reaction, $\mathrm{K}$
$\mathrm{T}_{\mathrm{g}, 0} \quad$ Gas phase's initial temperature, $\mathrm{K}$
$\mathrm{T}_{\mathrm{g}} \quad$ Gas phase's temperature, $\mathrm{K}$
$\mathrm{u}_{\mathrm{sg}} \quad$ Gas superficial velocity, $\mathrm{m} / \mathrm{s}$
$\mathrm{z} \quad$ Axial coordinate, $\mathrm{m}$

\section{Greek symbols}

$\varepsilon_{\mathrm{b}} \quad$ Packed-bed porosity, $\mathrm{m}^{3}$ of gas $/ \mathrm{m}^{3}$ of reformer

$\varepsilon_{\mathrm{p}} \quad$ Particle porosity, $\mathrm{m}^{3}$ of particle $/ \mathrm{m}^{3}$ of reformer

$\eta_{\mathrm{i}} \quad$ Effectiveness factors of reforming reactions $\mathrm{j}(\mathrm{j}=\mathrm{SRM}$ and WGSR)

$\lambda_{\mathrm{g}, \text { mix. }}$ Gaseous mixture's thermal conductivity, $\mathrm{W} / \mathrm{m} \mathrm{K}$

$\lambda_{\mathrm{g}, \text { eff. }} \quad$ Gas phase's effective thermal conductivity, $\mathrm{W} / \mathrm{m} \mathrm{K}$

$\lambda_{\mathrm{s}, \text { eff. }} \quad$ Solid phase's effective thermal conductivity, $\mathrm{W} / \mathrm{m} \mathrm{K}$

$\rho_{\mathrm{g}, \text { mix. }} \quad$ Gas mixture density of components $\mathrm{I}, \mathrm{kg} / \mathrm{m}^{3}$

$\rho_{\mathrm{s}} \quad$ Solid phase density, $\mathrm{kg} / \mathrm{m}^{3}$

\section{INTRODUCTION}

The production of hydrogen $\left(\mathrm{H}_{2}\right)$ can be carried out from different methods such as thermochemical (heat and chemical reactions to produce $\mathrm{H}_{2}$ ), 
reforming of hydrocarbons, biomass gasification, coal gasification, electrolytic, and biological (Lima et al., 2020; Anjos et al., 2020). The production of $\mathrm{H}_{2}$ in packed bed reformers is still attracting the interest of researchers and engineers. Up to date, several works have been published in the literature dealing with reforming method to produce $\mathrm{H}_{2}$ on different commercial catalyst (Silva and Abreu, 2017; Fuqiang et al., 2015; Yu et al., 2017). This work has as novelty the thermochemical conversion of $\mathrm{CH}_{4}$ on a $\beta$-SiC open-cell foam in a Thermochemical Packed Bed (TPB) reformer. $\beta$-SiC open-cell foams have been considered as potential candidates for catalyst support in the heterogeneous catalysis field due to their high external surface area combined with a low pressure drop (Chen et al., 2018). On the other hand, foam-based packed bed reformers offer great potential advantages for the thermal energy storage due to bed's high porosity.

The hydrodynamic characterization of the Steam Reforming of Methane (SRM) through $\beta$-SiC open-cell foam in a packed bed configuration in the TPB reformer is performed by reacting Methane $\left(\mathrm{CH}_{4}\right)$ with water steam $\left(\mathrm{H}_{2} \mathrm{O}\right)$. A physicalmathematical modelling is important to design and optimize the SRM method in the TPB reformer. Usually, the SRM method's application through $\beta$ $\mathrm{SiC}$ foam bed improves the heat transfer and mass transfer due to high porosity and surface area of the $\beta$-SiC foam (Vanhaecke et al., 2012). Mass, energy and momentum balance's equations for the gas phase as well as mass, energy and momentum balance's equations to the solid phase are described through mathematical models (Ma et al., 2016; Hussain et al., 2006). Here, a Non-isothermal Pseudo-Homogeneous Mathematical (NIPHM) model is used to model the SRM method in the TPB reformer. The NIPHM model is described by a system of Nonlinear Partial Differential Equations (NPDEs) in together with a developed kinetic model for reforming reactions.

With the purpose to reduce the research cost and project time, the mathematical modelling and computer simulation are extensively used to obtain a better understanding of design parameters in reformers. The approach and solution of physicalmathematical models are still a novelty of TPB reformers and thus the topic is a very actual in accessible literature. This work goal lies in the application of open-cell foam $(\beta-\mathrm{SiC})$ bed for the SRM method. A numerical analysis has been driven to investigate the molar flows of chemical components $\mathrm{i}$ on the SRM method of the TPB reformer. In addition, the profiles of the endothermic reaction temperature were also analyzed for the SRM method in the TPB reformer.

\section{PHYSICAL MODEL}

A schematic setup is used to study the thermochemical conversion of the SRM method in
TPB reformer according to Fig. 1. The physical model from TPB reformer is shown in Fig. 1.

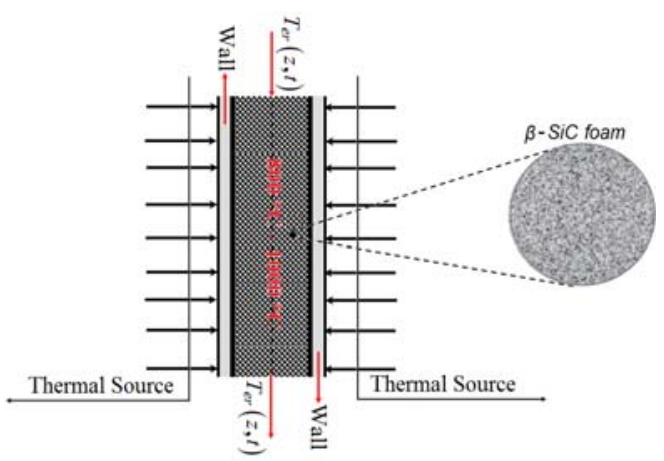

Figure 1. Schematic setup of the physical model from TPB reformer to study the SRM method on the $\beta$-SiC foam.

\section{KINETIC MODEL}

The reforming reaction of $\mathrm{CH}_{4}$ is used to produce syngas $\left(\mathrm{H}_{2}\right.$ e $\left.\mathrm{CO}\right)$ and it is highly endothermic (Cruz and Silva, 2017). On the other hand, the water-gas shift reaction (Eq. (1b)) is slightly exothermic and it is favoured at low temperatures. The SRM process has a limited equilibrium and can be described by the following reforming reaction:

$$
\begin{gathered}
\mathrm{SRM}: \mathrm{CH}_{4(\mathrm{~g})}+\mathrm{H}_{2} \mathrm{O}_{(\mathrm{g})} \leftrightarrow \mathrm{CO}_{(\mathrm{g})}+3 \mathrm{H}_{2(\mathrm{~g})} ; \\
\Delta \mathrm{H}_{298 \mathrm{~K}}^{0}=205,8 \mathrm{~kJ} / \mathrm{mol} \\
\text { WGSR : } \mathrm{CO}_{(\mathrm{g})}+\mathrm{H}_{2} \mathrm{O}_{(\mathrm{g})} \leftrightarrow \mathrm{CO}_{2(\mathrm{~g})}+\mathrm{H}_{2(\mathrm{~g})} ; \\
\Delta \mathrm{H}_{298 \mathrm{~K}}^{0}=-41,1 \mathrm{~kJ} / \mathrm{mol}
\end{gathered}
$$

As Eq. (1a) is a highly endothermic reaction, then it needs to high temperatures to obtain great $\mathrm{H}_{2}$ productivity. The overall rate equations of reactions, Eq. (1a) and Eq. (1b), are based on the LangmuirHinshelwood kinetic model and can be found in Ref. (Cruz and Silva, 2017). The kinetic rates from Eqs. (1a) and (1b) are considered more general for nickel (Ni) catalyst and, therefore, the equations of the SRM process is reported as:

$$
\begin{aligned}
& \mathrm{R}_{\mathrm{SRM}}=\frac{\frac{\mathrm{k}_{\mathrm{SRM}}}{\mathrm{P}_{\mathrm{H}_{2}}^{2.5}}\left(\mathrm{P}_{\mathrm{CH}_{4}} \mathrm{P}_{\mathrm{H}_{2} \mathrm{O}}-\frac{\mathrm{P}_{\mathrm{H}_{2}}^{3} \mathrm{P}_{\mathrm{CO}}}{\mathrm{K}_{\mathrm{SRM}}}\right)}{\alpha^{2}} \\
& \mathrm{R}_{\mathrm{WGSR}}=\frac{\frac{\mathrm{k}_{\mathrm{WGSR}}}{\mathrm{P}_{\mathrm{H}_{2}}}\left(\mathrm{P}_{\mathrm{CO}} \mathrm{P}_{\mathrm{H}_{2} \mathrm{O}}-\frac{\mathrm{P}_{\mathrm{H}_{2}} \mathrm{P}_{\mathrm{CO}_{2}}}{\mathrm{~K}_{\mathrm{WGSR}}}\right)}{\alpha^{2}}
\end{aligned}
$$

where, 


$$
\begin{aligned}
\alpha= & 1+\frac{\mathrm{F}_{\mathrm{H}_{2} \mathrm{O}, 0}}{\mathrm{~F}_{\mathrm{CH}_{4}, 0}}+\frac{\mathrm{F}_{\mathrm{H}_{2}, 0}}{\mathrm{~F}_{\mathrm{CH}_{4}, 0}}+\mathrm{K}_{\mathrm{CO}} \mathrm{P}_{\mathrm{CO}}+\mathrm{K}_{\mathrm{H}_{2}} \mathrm{P}_{\mathrm{H}_{2}} \\
& +\mathrm{K}_{\mathrm{CH}_{4}} \mathrm{P}_{\mathrm{CH}_{4}}+\frac{\mathrm{K}_{\mathrm{H}_{2} \mathrm{O}} \mathrm{P}_{\mathrm{H}_{2} \mathrm{O}}}{\mathrm{P}_{\mathrm{H}_{2}}}
\end{aligned}
$$

The partial pressures of chemical components $\mathrm{i}$, $\mathrm{i}=\mathrm{CH}_{4}, \mathrm{H}_{2} \mathrm{O}, \mathrm{CO}, \mathrm{CO}_{2}$ and $\mathrm{H}_{2}$ from Eqs. (2a)-(2c), are computed using Eqs. (3a)-(3e) as follows.

$$
\begin{aligned}
& \mathrm{P}_{\mathrm{CH}_{4}}=\frac{1-\left(1-\frac{\mathrm{F}_{\mathrm{CH}_{4}}}{\mathrm{~F}_{\mathrm{CH}_{4}, 0}}\right)}{1+\sum_{\mathrm{j}}^{4} \frac{\mathrm{F}_{\mathrm{j}, 0}}{\mathrm{~F}_{\mathrm{CH}_{4}, 0}}} \mathrm{P}_{\mathrm{op}} . \\
& \mathrm{P}_{\mathrm{H}_{2} \mathrm{O}}=\frac{\frac{\mathrm{F}_{\mathrm{H}_{2} \mathrm{O}, 0}}{\mathrm{~F}_{\mathrm{CH}_{4}, 0}}-\left(1-\frac{\mathrm{F}_{\mathrm{CH}_{4}}}{\mathrm{~F}_{\mathrm{CH}_{4}, 0}}\right)-\left(\frac{\mathrm{F}_{\mathrm{CO}_{2}}}{\mathrm{~F}_{\mathrm{CH}_{4}, 0}}\right)}{1+\sum_{\mathrm{j}}^{4} \frac{\mathrm{F}_{\mathrm{j}, 0}}{\mathrm{~F}_{\mathrm{CH}_{4}, 0}}} \mathrm{P}_{\mathrm{op}} . \\
& \mathrm{P}_{\mathrm{CO}}=\frac{\frac{\mathrm{F}_{\mathrm{CO}, 0}}{\mathrm{~F}_{\mathrm{CH}_{4}, 0}}+\left(1-\frac{\mathrm{F}_{\mathrm{CH}_{4}}}{\mathrm{~F}_{\mathrm{CH}_{4}, 0}}\right)-\left(\frac{\mathrm{F}_{\mathrm{CO}_{2}}}{\mathrm{~F}_{\mathrm{CH}_{4}, 0}}\right)}{1+\sum_{\mathrm{j}}^{4} \frac{\mathrm{F}_{\mathrm{j}, 0}}{\mathrm{~F}_{\mathrm{CH}_{4}, 0}}} \mathrm{P}_{\mathrm{op}} . \\
& \mathrm{P}_{\mathrm{CO}_{2}}=\frac{\frac{\mathrm{F}_{\mathrm{CO}_{2}, 0}}{\mathrm{~F}_{\mathrm{CH}_{4}, 0}}+\left(\frac{\mathrm{F}_{\mathrm{CO}_{2}}}{\mathrm{~F}_{\mathrm{CH}_{4}, 0}}\right)}{1+\sum_{\mathrm{j}}^{4} \frac{\mathrm{F}_{\mathrm{j}, 0}}{\mathrm{~F}_{\mathrm{CH}_{4}, 0}}} \mathrm{P}_{\text {op. }} \text {. } \\
& \mathrm{P}_{\mathrm{H}_{2}}=\frac{\frac{\mathrm{F}_{\mathrm{H}_{2}, 0}}{\mathrm{~F}_{\mathrm{CH}_{4}, 0}}+3\left(1-\frac{\mathrm{F}_{\mathrm{CH}_{4}}}{\mathrm{~F}_{\mathrm{CH}_{4}, 0}}\right)-\left(\frac{\mathrm{F}_{\mathrm{CO}_{2}}}{\mathrm{~F}_{\mathrm{CH}_{4}, 0}}\right)-\frac{\mathrm{F}_{\mathrm{H}_{2}}}{\mathrm{~F}_{\mathrm{CH}_{4}, 0}}}{1+\sum_{\mathrm{j}}^{4} \frac{\mathrm{F}_{\mathrm{j}, 0}}{\mathrm{~F}_{\mathrm{CH}_{4}, 0}}} \mathrm{P}_{\text {op. }} . \\
& \mathrm{j}=\mathrm{H}_{2} \mathrm{O}, \mathrm{CO}, \mathrm{CO}_{2} \text { and } \mathrm{H}_{2}
\end{aligned}
$$

For the purpose of obtaining the net rates $\left(\mathrm{r}_{\mathrm{i}}\right)$ to each chemical component $\mathrm{i}\left(\mathrm{i}=\mathrm{CH}_{4}, \mathrm{H}_{2} \mathrm{O}, \mathrm{CO}, \mathrm{CO}_{2}\right.$ and $\mathrm{H}_{2}$ ), an expression is used to compute these net rates as follows.

$$
\mathrm{r}_{\mathrm{i}}=\sum_{\mathrm{i}=1}^{5} \sum_{\mathrm{j}=1}^{2} \eta_{\mathrm{j}} \sigma_{\mathrm{ij}} \mathrm{R}_{\mathrm{j}} ; \mathrm{j}=\text { SRM, WGSR }
$$

In relation to the net rates, Eq. (4) is used to describe the net rate of each chemical component $i$ of Eqs. (1a) and (1b) as follows.

$$
\begin{gathered}
\mathrm{r}_{\mathrm{CH}_{4}}=-\eta_{\mathrm{SRM}} \mathrm{R}_{\mathrm{SRM}} \\
\mathrm{r}_{\mathrm{H}_{2} \mathrm{O}}=-\left(\eta_{\mathrm{SRM}} \mathrm{R}_{\mathrm{SRM}}+\eta_{\mathrm{WGSR}} \mathrm{R}_{\mathrm{WGSR}}\right) \\
\mathrm{r}_{\mathrm{CO}}=\eta_{\mathrm{SRM}} \mathrm{R}_{\mathrm{SRM}}-\eta_{\mathrm{WGSR}} \mathrm{R}_{\mathrm{WGSR}} \\
\mathrm{r}_{\mathrm{H}_{2}}=3 \eta_{\mathrm{SRM}} \mathrm{R}_{\mathrm{SRM}}+\eta_{\mathrm{WGSR}} \mathrm{R}_{\mathrm{WGSR}}
\end{gathered}
$$

$$
\mathrm{r}_{\mathrm{CO}_{2}}=\eta_{\mathrm{WGSR}} \mathrm{R}_{\mathrm{WGSR}}
$$

The kinetic constants, equilibrium constants and adsorption equilibrium constants for the calculations of reaction rates are described as a function of operating temperature (Cruz and Silva, 2017).

$$
\begin{gathered}
\mathrm{k}_{\mathrm{SRM}}=2.636 \times 10^{13} \exp \left(-\frac{240.10}{\mathrm{R}_{\mathrm{u}} \mathrm{T}_{\text {op. }}}\right) \\
\mathrm{K}_{\mathrm{SRM}}=1.0267 \times 10^{4} \exp \left(-\frac{26830.0}{\mathrm{~T}_{\mathrm{op} .}}\right)+30.114 \\
\mathrm{k}_{\mathrm{WGSR}}=12.194 \exp \left(-\frac{240.10}{\mathrm{R}_{\mathrm{u}} \mathrm{T}_{\text {op. }}}\right) \\
\mathrm{K}_{\mathrm{WGSR}}=\exp \left(-\frac{26830.0}{\mathrm{~T}_{\mathrm{op}}}\right)-4.063 \\
\mathrm{~K}_{\mathrm{CH}_{4}}=6.650 \times 10^{-6} \exp \left(-\frac{38.28}{\mathrm{R}_{\mathrm{u}} \mathrm{T}_{\text {op. }}}\right) \\
\mathrm{K}_{\mathrm{H}_{2} \mathrm{O}}=1.770 \times 10^{5} \exp \left(-\frac{88.68}{\mathrm{R}_{\mathrm{u}} \mathrm{T}_{\text {op. }}}\right) \\
\mathrm{K}_{\mathrm{H}_{2}}=6.120 \times 10^{-11} \exp \left(\frac{82.90}{\mathrm{R}_{\mathrm{u}} \mathrm{T}_{\text {op. }}}\right) \\
\mathrm{K}_{\mathrm{CO}}=8.230 \times 10^{-7} \exp \left(\frac{70.65}{\mathrm{R}_{\mathrm{u}} \mathrm{T}_{\text {op. }}}\right)
\end{gathered}
$$

\section{MATHEMATICAL MODELLLING}

The thermochemical modelling from TPB reformer are described by a NIPHM model. The development of the NIPHM model takes into account the following assumptions: (1) the NIPHM model is described under non-isothermal condition, (2) the NIPHM model from TPB reformer is plug-flow with axial dispersion under transient condition, (3) the radial dispersion is negligible inside TPB reformer, (4) the gaseous mixture has constant density in TPB reformer, (5) the molar flow rates inside TPB reformer are constant, (6) the deposition effect of carbon at the surface of catalytic particles has been neglected, (7) the gas behavior inside TPB reformer was considered as an ideal gas mixture, (8) the bed porosity in axial direction is considered constant, (9) the heaviest hydrocarbons than methane have not been considered in this work, and (10) chemical reactions as assumed to take place at the surface of catalyst particles. These premises are used to build the governing equations of the NIPHD model inside TPB reformer as follows.

\section{GAS PHASE'S ENERGY BALANCE}

A governing equation is described to study the heat distribution on the gas phase inside TPB 
reformer. The proposed equation provides clear information to drive the temperature distribution on the gas phase under open-cell foam $(\beta-\mathrm{SiC})$ bed. Therefore, a one-dimensional dynamic equation is developed for the gas phase's temperature as follows.

$\rho_{\mathrm{g}, \text { mix. }} \mathrm{C}_{\mathrm{p}, \mathrm{g}, \text { mix. }}\left(\frac{\partial \mathrm{T}_{\mathrm{g}}}{\partial \mathrm{t}}+\frac{4 \mathrm{q}_{\mathrm{g}}}{\pi \mathrm{d}_{\text {refor. }}^{2}} \frac{\partial \mathrm{T}_{\mathrm{g}}}{\partial \mathrm{z}}\right)=$

$\lambda_{\mathrm{g}, \mathrm{eff}} \frac{\partial^{2} \mathrm{~T}_{\mathrm{g}}}{\partial \mathrm{z}^{2}}-\mathrm{h}_{\mathrm{gs}} \frac{\left(1-\varepsilon_{\mathrm{b}}\right)}{\varepsilon_{\mathrm{b}}} \frac{6}{\mathrm{~d}_{\mathrm{p}}}\left(\mathrm{T}_{\mathrm{g}}-\mathrm{T}_{\mathrm{er}}\right)$

The effective thermal conductivity of the gas phase is defined as a function of the gas thermal conductivity as follows.

$$
\begin{gathered}
\lambda_{\mathrm{g}, \text { eff. }}=\varepsilon_{\mathrm{b}} \lambda_{\mathrm{g}, \text { mix }} . \\
\lambda_{\mathrm{g}, \text { mix. }}=1.52 \times 10^{-11} \mathrm{~T}_{\mathrm{g}, 0}^{3}-4.86 \times 10^{-8} \mathrm{~T}_{\mathrm{g}, 0}^{2}+ \\
1.02 \times 10^{-4} \mathrm{~T}_{\mathrm{g}, 0}-3.93 \times 10^{-3}
\end{gathered}
$$

The suitable initial and boundary conditions from Eq. (7a) are given as follows.

$$
\begin{gathered}
\left.\mathrm{T}_{\mathrm{g}}\right|_{\mathrm{t}=0}=\mathrm{T}_{\mathrm{g}, 0} ; 0 \leq \mathrm{z} \leq \mathrm{L} \\
\left.\frac{\partial \mathrm{T}}{\partial \mathrm{z}}\right|_{\mathrm{z}=0^{+}}=\frac{\rho_{\mathrm{g}, \text { mix. }} \mathrm{C}_{\mathrm{p}, \mathrm{g}, \text { mix. }}}{\lambda_{\mathrm{g}, \text { mix. }}} \frac{4 \mathrm{q}_{\mathrm{g}}}{\pi \mathrm{d}_{\text {ref. }}^{2}} \\
\frac{\left.\partial \mathrm{T}_{\mathrm{g}}\right|_{\mathrm{z}=\mathrm{L}}=}{\left.\left.\mathrm{T}_{\mathrm{g}}\right|_{\mathrm{z}=0^{+}}-\mathrm{T}_{\mathrm{g}, \infty}\right) ; \mathrm{t} \geq 0} \\
\lambda_{\mathrm{g}, \text { eff. }}
\end{gathered}
$$

\section{SOLID PHASE'S ENERGY BALANCE}

The reactive packed-bed has been filled with $\beta$ $\mathrm{SiC}$ open-cell foams. The energy balance equation is built at the surface of $\beta$-SiC open-cell foams where take place the endothermic reaction of $\mathrm{CH}_{4}$. The thermal storage takes place on the $\beta$-SiC open-cell foams to ensure sufficient energy to process the SRM's endothermic reaction. However, the energy balance for the SRM's temperature at the surface of $\beta$-SiC open-cell foams is given as follows.

$$
\begin{gathered}
\rho_{\mathrm{s}} \mathrm{C}_{\mathrm{p}, \mathrm{s}} \frac{\partial \mathrm{T}_{\text {er }}}{\partial \mathrm{t}}=\lambda_{\mathrm{s}, \text { eff }} \frac{\partial^{2} \mathrm{~T}_{\text {er }}}{\partial \mathrm{z}^{2}}+\mathrm{h}_{\mathrm{sg}} \frac{6}{\mathrm{~d}_{\mathrm{p}}} \frac{\left(1-\varepsilon_{\mathrm{b}}\right)}{\varepsilon_{\mathrm{b}}} \\
\left(\mathrm{T}_{\mathrm{g}}-\mathrm{T}_{\mathrm{er}}\right)+\rho_{\mathrm{s}} \frac{\left(1-\varepsilon_{\mathrm{p}}\right)}{\varepsilon_{\mathrm{p}}} \sum_{\mathrm{j}=1}^{2} \pm \Delta \mathrm{H}_{\mathrm{j}} \eta_{\mathrm{j}} \mathrm{R}_{\mathrm{j}}
\end{gathered}
$$

The solid phase's thermal conductivity is defined as a function of the gaseous mixture's thermal conductivity according to Eq. (8b).

$$
\lambda_{\mathrm{s}, \mathrm{eff}}=\left(1-\varepsilon_{\mathrm{b}}\right) \lambda_{\mathrm{g}, \mathrm{mix}}
$$

The suitable initial and boundary conditions from Eq. (8a) are given as follows.

$$
\begin{aligned}
& \left.\mathrm{T}_{\mathrm{er}}\right|_{\mathrm{t}=0}=\mathrm{T}_{\mathrm{er}, 0} ; 0 \leq \mathrm{z} \leq \mathrm{L} \\
& \left.\frac{\partial \mathrm{T}_{\mathrm{er}}}{\partial \mathrm{z}}\right|_{\mathrm{z}=0^{+}}=\frac{\mathrm{q}_{\mathrm{h}}}{\lambda_{\mathrm{s}}} ; \mathrm{t} \geq 0
\end{aligned}
$$

$\left.\frac{\partial \mathrm{T}_{\mathrm{er}}}{\partial \mathrm{z}}\right|_{\mathrm{z}=\mathrm{L}}=\frac{\mathrm{h}_{\mathrm{sg}, \text { eff }}}{\lambda_{\mathrm{s}, \text { eff. }}}\left(\mathrm{T}_{\mathrm{er}, \infty}-\left.\mathrm{T}_{\mathrm{er}}\right|_{\mathrm{z}=\mathrm{L}}\right) ; \mathrm{t} \geq 0$

\section{TRANSPORT EQUATION OF CHEMICAL COMPONENTS}

In Section TPB reformer's mathematical modelling, it was approached some assumptions that can be used to build the proposed problem's modelling. However, the transport equations for chemical components $\mathrm{i}\left(\mathrm{i}=\mathrm{CH}_{4}, \mathrm{H}_{2} \mathrm{O}, \mathrm{CO}, \mathrm{CO}\right.$ and $\mathrm{H}_{2}$ ) are presented as follows.

$$
\begin{array}{r}
\frac{\mathrm{u}_{\mathrm{sg}}}{\mathrm{g}} \frac{\partial \mathrm{F}_{\mathrm{i}}}{\partial \mathrm{t}}+\frac{4 \mathrm{q}_{\mathrm{g}}}{\mathrm{S}_{\mathrm{sp}} \pi \mathrm{d}_{\text {ref. }}^{2}} \frac{\partial \mathrm{F}_{\mathrm{i}}}{\partial \mathrm{z}}=\frac{\mathrm{D}_{\mathrm{ax}, \mathrm{i}}}{\mathrm{S}_{\mathrm{sp}}} \frac{\partial^{2} \mathrm{~F}_{\mathrm{i}}}{\partial \mathrm{z}^{2}}+\rho_{\mathrm{s}} \mathrm{L} \\
\mathrm{r}_{\text {ref. }}^{2}\left(1-\varepsilon_{\mathrm{b}}\right) \mathrm{r}_{\mathrm{i}} ; 0<\mathrm{z}<\mathrm{L}
\end{array}
$$

The suitable initial and boundary conditions for Eq. (9a) are presented as follows.

$$
\begin{gathered}
\left.\mathrm{F}_{\mathrm{i}}\right|_{\mathrm{t}=0,}=\mathrm{F}_{\mathrm{i}, 0} ; 0 \leq \mathrm{z} \leq \mathrm{L} \\
\left.\frac{\partial \mathrm{F}_{\mathrm{i}}}{\partial \mathrm{z}}\right|_{\mathrm{z}=0^{+}}=\frac{\mathrm{u}_{\mathrm{sg}} \mathrm{L}}{\varepsilon_{\mathrm{b}} \mathrm{D}_{\mathrm{ax}, \mathrm{i}}}\left(\left.\mathrm{F}_{\mathrm{i}}\right|_{\mathrm{z}=0^{+}}-\mathrm{F}_{\mathrm{i}, \infty}\right) ; \mathrm{t} \geq 0 \\
\left.\frac{\partial \mathrm{F}_{\mathrm{i}}}{\partial \mathrm{z}}\right|_{\mathrm{z}=\mathrm{L}}=\frac{\mathrm{k}_{\mathrm{gs}, \mathrm{eff}} \mathrm{L}}{\varepsilon_{\mathrm{b}} \mathrm{D}_{\mathrm{ax}, \mathrm{i}}}\left(\left.\mathrm{F}_{\mathrm{i}}\right|_{\mathrm{z}=\mathrm{L}}-\mathrm{F}_{\mathrm{i}, \infty}\right) ; \mathrm{t} \geq 0
\end{gathered}
$$

\section{PROPOSED MODEL'S SOLUTION BY CIEA}

The CIEA's method can be employed as a good technique for studying the performance from TPB reformers (Dias and Silva, 2020). The CIEA's methodology has been used to transform the PDE system (Eqs. (7a), (8a), and (9a)) into an ODE system using the boundary conditions (Eqs. (7e), (7f), (8d), (8e), (9c) and (9d)) of each PDE. After transforming of PDEs into ODEs, the system of ODEs is reported as follows. 


$$
\begin{aligned}
& \alpha_{\mathrm{g}, 1} \frac{\mathrm{dT}_{\mathrm{g}}(\mathrm{t})}{\mathrm{dt}}=\left(\alpha_{\mathrm{g}, 2}-\lambda_{\mathrm{g}, \text { eff. }} \alpha_{\mathrm{g}, 4}\right) \mathrm{T}_{\mathrm{g}}(0, \mathrm{t})- \\
&\left(\alpha_{\mathrm{g}, 2}+\lambda_{\mathrm{g}, \text { eff. }} \alpha_{\mathrm{g}, 5}\right) \mathrm{T}_{\mathrm{g}}(\mathrm{L}, \mathrm{t})- \\
& \alpha_{\mathrm{g}, 3}\left(\overline{\mathrm{T}}_{\mathrm{g}}(\mathrm{t})-\overline{\mathrm{T}}_{\mathrm{s}}(\mathrm{t})\right)+\lambda_{\mathrm{g}, \mathrm{eff}} \\
& \mathrm{T}_{\mathrm{g}, \infty}\left(\alpha_{\mathrm{g}, 4}+\alpha_{\mathrm{g}, 5}\right) \\
& \frac{\mathrm{dT} \overline{\mathrm{er}}_{\mathrm{r}}(\mathrm{t})}{\mathrm{dt}}=\beta_{\mathrm{s}, 5}\left(\overline{\mathrm{T}}_{\mathrm{g}}(\mathrm{t})-\overline{\mathrm{T}}_{\mathrm{er}}(\mathrm{t})\right)-\beta_{\mathrm{s}, 1} \beta_{\mathrm{s}, 5} \\
& \mathrm{~T}_{\mathrm{er}}(\mathrm{L}, \mathrm{t})+\beta_{\mathrm{s}, 1}\left(\beta_{\mathrm{s}, 5} \mathrm{~T}_{\mathrm{er}, \infty}-\beta_{\mathrm{s}, 4}\right) \\
&+\sum_{\mathrm{j}=1}^{2} \pm \Delta \mathrm{H}_{\mathrm{j}} \eta_{\mathrm{j}} \overline{\mathrm{R}}_{\mathrm{j}}(\mathrm{t}) \\
& \frac{\mathrm{dF}_{\mathrm{i}}(\mathrm{t})}{\mathrm{dt}}=\left(\varphi_{\mathrm{f}, 2} \varphi_{\mathrm{f}, 5}-\varphi_{\mathrm{f}, 1}\right) \mathrm{F}_{\mathrm{i}}(\mathrm{L}, \mathrm{t})-\mathrm{F}_{\mathrm{i}}(0, \mathrm{t}) \\
&\left(\varphi_{\mathrm{f}, 2} \varphi_{\mathrm{f}, 4}-\varphi_{\mathrm{f}, 1}\right)+\varphi_{\mathrm{f}, 3} \overline{\mathrm{F}}_{\mathrm{i}}(\mathrm{t})+\mathrm{F}_{\mathrm{i}, \infty} \\
& \varphi_{\mathrm{f}, 2}\left(\varphi_{\mathrm{f}, 4}-\varphi_{\mathrm{f}, 5}\right)
\end{aligned}
$$

All coefficients from Eqs. (10a)-(10c) are presented in Appendices A, B and C, respectively.

\section{FULL SOLUTION}

The numeric methodology's choice is limited to the desired accuracy on the consistency and robustness of PDE system's numerical data (Cruz and Silva, 2017). In relation to ODEs of this work, Eqs. (10a) - (10c), they were solved by Runge-Kutta Gill method. On the other hand, the full solutions are approximated as follows.

$$
\begin{aligned}
\mathrm{T}_{\mathrm{g}}(\mathrm{z}, \mathrm{t})= & \left.\frac{1}{2} \mathrm{~T}_{\mathrm{g}}(\mathrm{z}, \mathrm{t})\right|_{\mathrm{t}=0}+\sum_{\mathrm{k}=1}^{\infty} \overline{\mathrm{T}}_{\mathrm{g}}\left(\mathrm{t}_{\mathrm{k}}\right) \\
& \sin \left(\frac{\mathrm{k} \pi \mathrm{z}}{\mathrm{L}}\right) \\
\mathrm{T}_{\text {er }}(\mathrm{z}, \mathrm{t})= & \left.\frac{1}{2} \mathrm{~T}_{\mathrm{er}}(\mathrm{z}, \mathrm{t})\right|_{\mathrm{t}=0}+\sum_{\mathrm{k}=1}^{\infty} \overline{\mathrm{T}}_{\mathrm{er}}\left(\mathrm{t}_{\mathrm{k}}\right) \\
& \sin \left(\frac{\mathrm{k} \pi \mathrm{z}}{\mathrm{L}}\right) \\
\mathrm{F}_{\mathrm{i}}(\mathrm{z}, \mathrm{t})= & \left.\frac{1}{2} \mathrm{~F}_{\mathrm{i}}(\mathrm{z}, \mathrm{t})\right|_{\mathrm{t}=0}+\sum_{\mathrm{k}=1}^{\infty} \overline{\mathrm{F}}_{\mathrm{i}}\left(\mathrm{t}_{\mathrm{k}}\right) \\
& \sin \left(\frac{\mathrm{k} \pi \mathrm{z}}{\mathrm{L}}\right)
\end{aligned}
$$

\section{RESULTS AND DISCUSSIONS}

In the mathematical model, a physicalmathematical modelling has been developed to investigate the SRM method in the TPB reformer. A computational algorithm using the FORTRAN 95 has been elaborated by authors to compute the SRM method's variables in the TPB reformer.

\section{KINETIC PARAMETERS}

In section of the physical model of this work, kinetic model's equations were presented. The kinetic model's parameters are used to feed the computational algorithm in combination with hydrodynamic model's parameters from the TPB reformer. The numerical values of the kinetic model's parameters are shown in Table 1 below:

Table 1. Kinetic constants of reactions, equilibrium constants of reactions and adsorption constants

\begin{tabular}{lll}
\hline Parameters & Values & Sources \\
\hline $\begin{array}{l}\mathrm{k}_{\mathrm{SRM}}\left(\mathrm{mol}(\mathrm{kPa})^{0.5}\right. \\
/ \mathrm{kgcat}^{(1)}\end{array}$ & $2.015 \times 10^{11}$ & Eq. $(6 \mathrm{a})$ \\
$\mathrm{K}_{\mathrm{SRM}}(\mathrm{kPa})^{2(1)}$ & $2.135 \times 10^{7}$ & Eq. $(6 \mathrm{~b})$ \\
$\mathrm{K}_{\mathrm{WGSR}}(\mathrm{mol}$ & $1.218 \times 10^{3}$ & Eq. $(6 \mathrm{c})$ \\
$\left.(\mathrm{kPa})^{-1} / \mathrm{kg}_{\text {cat. }}\right)^{(1)}$ & & \\
$\mathrm{K}_{\mathrm{WGSR}}(-)^{(1)}$ & 13.015 & Eq. $(6 \mathrm{~d})$ \\
$\mathrm{K}_{\mathrm{CH} 4}(\mathrm{kPa})^{-1}(1)$ & $8.974 \times 10^{-7}$ & Eq. $(6 \mathrm{e})$ \\
$\mathrm{K}_{\mathrm{H} 2 \mathrm{O}}(-)^{(1)}$ & $3.701 \times 10^{4}$ & Eq. $(6 \mathrm{f})$ \\
$\mathrm{K}_{\mathrm{H} 2}(\mathrm{kPa})^{-1}(1)$ & $8.987 \times 10^{-12}$ & Eq. $(6 \mathrm{~g})$ \\
$\mathrm{K}_{\mathrm{CO}}(\mathrm{kPa})^{-1(1)}$ & $5.671 \times 10^{-6}$ & Eq. $(6 \mathrm{~h})$ \\
$\eta_{\mathrm{SRM}}(-)^{(1)}$ & 0.021 & Cruz and Silva, \\
$\eta_{\text {WGSR }}(-)^{(1)}$ & 0.0169 & 2017 \\
\end{tabular}

${ }^{(1)}$ Computed at $900^{\circ} \mathrm{C}$

\section{MODEL PARAMETERS}

In Tables 2 and 3, operating conditions and hydrodynamics parameters have been presented. Table 2 shows the operating conditions of TPB reformer as well as bed properties of this reformer On the other hand, Table 3 reports thermophysical properties, inlet molar flow of components and dispersion coefficients of chemical components.

Table 2. Operating conditions and bed properties for the TPB reformer.

\begin{tabular}{lll}
\hline Model parameters & Values & Source \\
\hline $\mathrm{L}(\mathrm{m})$ & 0.60 & Estimated \\
$\mathrm{d}_{\mathrm{p}}(\mathrm{m})$ & $3.4 \times 10^{-4}$ & Estimated \\
$\mathrm{d}_{\mathrm{refor}}(\mathrm{m})$ & $3.2 \times 10^{-3}$ & Estimated \\
$\mathrm{T}_{\mathrm{g}, 0}(\mathrm{~K})$ & 673 & Estimated \\
$\mathrm{T}_{\mathrm{er}, 0}(\mathrm{~K})$ & 673 & Estimated \\
$\mathrm{P}_{\mathrm{op} .}(\mathrm{kPa})$ & 600 & Estimated \\
$\mathrm{q}_{\mathrm{g}}\left(\mathrm{m}^{3} / \mathrm{s}\right)$ & $1.201 \times 10^{-6}$ & Estimated \\
$\mathrm{g}\left(\mathrm{m} / \mathrm{s}^{2}\right)$ & 9.81 & Estimated \\
$\mathrm{Sp}\left(\mathrm{s}^{-1}\right)$ & $3.53 \times 10^{7}$ & Cruz and Silva, \\
$\varepsilon_{\mathrm{b}}\left(\mathrm{m}^{3}\right.$ of gas $/ \mathrm{m}^{3}$ of & 0.39 & Dias and Silva, \\
reformer $)$ & & 2020 \\
$\varepsilon_{\mathrm{p}}\left(\mathrm{m}^{3}\right.$ of gas $/ \mathrm{m}^{3}$ of & \multirow{2}{*}{0.87} & Voltolina et al., \\
reformer $)$ & & 2017 \\
\hline
\end{tabular}


Table 3. Thermophysical properties, inlet molar flow of components and dispersion parameters for the SRM process.

\begin{tabular}{|c|c|c|}
\hline Model parameters & Values & Source \\
\hline$\rho_{g}$, mix. $\left(\mathrm{kg} / \mathrm{m}^{3}\right)$ & 0.1692 & $\begin{array}{l}\text { Silva and } \\
\text { Abreu, } 2016\end{array}$ \\
\hline $\mathrm{C}_{\mathrm{p}, \mathrm{g}, \text { mix. }}(\mathrm{kJ} / \mathrm{kg} \mathrm{K})$ & 127.09 & $\begin{array}{l}\text { Dias and Silva, } \\
2020\end{array}$ \\
\hline$\lambda_{\mathrm{g}, \mathrm{eff}}(\mathrm{W} / \mathrm{m} \mathrm{K})$ & 1.078 & Eq. $(7 b)$ \\
\hline $\mathrm{h}_{\mathrm{gs}}\left(\mathrm{W} / \mathrm{m}^{2} \mathrm{~K}\right)$ & 1.902 & $\begin{array}{l}\text { Dias and Silva, } \\
2020\end{array}$ \\
\hline $\mathrm{C}_{\mathrm{p}, \mathrm{s}}(\mathrm{J} / \mathrm{kg} \mathrm{K})$ & 336.00 & $\begin{array}{l}\text { Dias and Silva, } \\
2020\end{array}$ \\
\hline$\lambda_{\mathrm{s}, \text { eff }}(\mathrm{W} / \mathrm{m} \mathrm{K})$ & 0.501 & Eq. $(6 b)$ \\
\hline $\mathrm{F}_{\mathrm{CH} 4,0}(\mathrm{~mol} / \mathrm{s})$ & 0.25 & $\begin{array}{l}\text { Silva and } \\
\text { Abreu, } 2016\end{array}$ \\
\hline $\mathrm{F}_{\mathrm{H} 2 \mathrm{O}, 0}(\mathrm{~mol} / \mathrm{s})$. & 0.75 & $\begin{array}{l}\text { Silva and } \\
\text { Abreu, } 2016\end{array}$ \\
\hline $\mathrm{F}_{\mathrm{H} 2,0}(\mathrm{~mol} / \mathrm{s})$ & $7.62 \times 10^{-5}$ & $\begin{array}{l}\text { Silva and } \\
\text { Abreu, } 2016\end{array}$ \\
\hline $\mathrm{F}_{\mathrm{CO}, 0}(\mathrm{~mol} / \mathrm{s})$ & $3.2 \times 10^{-5}$ & $\begin{array}{l}\text { Silva and } \\
\text { Abreu, } 2016\end{array}$ \\
\hline $\mathrm{F}_{\mathrm{CO} 2,0}(\mathrm{~mol} / \mathrm{s})$ & $6.32 \times 10^{-5}$ & $\begin{array}{l}\text { Silva and } \\
\text { Abreu, } 2016\end{array}$ \\
\hline $\mathrm{D}_{\mathrm{ax}, \mathrm{CH} 4}\left(\mathrm{~m}^{2} / \mathrm{s}\right)^{(1)}$ & $289 \times 10^{-3}$ & $\begin{array}{l}\text { Cruz and Silva, } \\
2017\end{array}$ \\
\hline $\mathrm{D}_{\mathrm{ax}, \mathrm{H} 2 \mathrm{O}}\left(\mathrm{m}^{2} / \mathrm{s}\right)^{(1)}$ & $3.79 \times 10^{-3}$ & $\begin{array}{l}\text { Cruz and Silva, } \\
2017\end{array}$ \\
\hline $\mathrm{D}_{\mathrm{ax}, \mathrm{H} 2}\left(\mathrm{~m}^{2} / \mathrm{s}\right)^{(1)}$ & $2.01 \times 10^{-3}$ & $\begin{array}{l}\text { Cruz and Silva, } \\
2017\end{array}$ \\
\hline $\mathrm{D}_{\mathrm{ax}, \mathrm{CO}}\left(\mathrm{m}^{2} / \mathrm{s}\right)^{(1)}$ & $3.41 \times 10^{-3}$ & $\begin{array}{l}\text { Cruz and Silva, } \\
2017\end{array}$ \\
\hline $\mathrm{D}_{\mathrm{ax}, \mathrm{CO} 2}\left(\mathrm{~m}^{2} / \mathrm{s}\right)^{(1)}$ & $1.89 \times 10^{-3}$ & $\begin{array}{l}\text { Cruz and Silva, } \\
2017\end{array}$ \\
\hline
\end{tabular}

(1) Computed at $900^{\circ} \mathrm{C}$

\section{VALIDATION AGAINST PUBLISHED DATA}

To ensure the validity of the proposed model, authors have made a comparison of simulated results with published data of the literature. The simulating results are computed from a computer code developed by authors. Slight differences can be found due to the deviation between the literature results and simulating results. An average relative error (ARE), Eq. (12a), was used to compute the consistency criterion and therefore, the ARE is given as follows.

$$
\begin{aligned}
& \mathrm{ARE}=\left|\frac{\mathrm{F}_{\mathrm{i}}^{\text {Exp. results }}-\mathrm{F}_{\mathrm{i}}^{\text {Simul. results }}}{\mathrm{F}_{\mathrm{i}}^{\text {Exp. results }}}\right| \times 100 ; \\
& \mathrm{i}=\mathrm{CH}_{4}, \mathrm{H}_{2} \mathrm{O}, \mathrm{H}_{2}, \mathrm{CO} \text { and } \mathrm{CO}_{2}
\end{aligned}
$$

Fig. 2 compares the literature results and simulated results of molar flows of chemical components $i$ at the outlet of the TPB reformer. The good agreement between the simulated results and literature data reports that the developed model is acceptable. As it can be seen in Fig. 2, the simulated results against the experimental results from literature (Chompupun et al., 2018) have presented a good agreement, resulting in AREs of $\mathrm{ARE}_{\mathrm{CH} 4}=9.28 \%$, $\mathrm{ARE}_{\mathrm{H} 2 \mathrm{O}}=0.81 \%, \mathrm{ARE}_{\mathrm{H} 2}=1.29 \%, \mathrm{ARE}_{\mathrm{CO}}=9.74 \%$, and $\mathrm{ARE}_{\mathrm{CO} 2}=2.13 \%$. These AREs should be due to the incompatibility between the simulating results and experimental results.

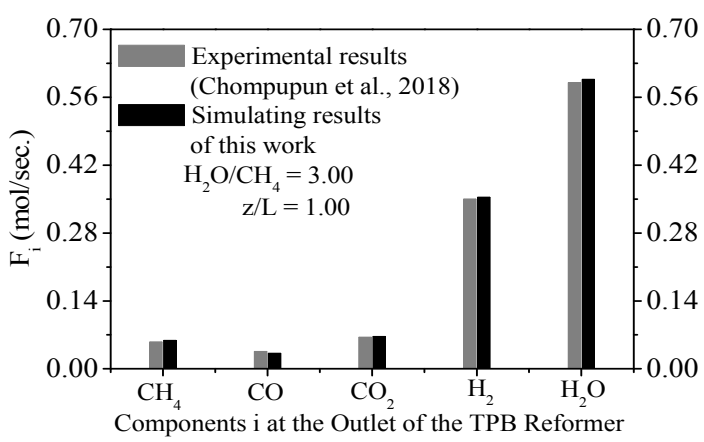

Figure 2. Comparison between molar flows of chemical components $i$ at the outlet of TPB reformer.

\section{NUMERICAL EXPERIMENTS}

The profiles of the Endothermic Reaction Temperature (ERT) were computed from Eq. (20) for the SRM method in the TPB reformer. In Fig. 3, it is checked the dynamic region and stationary region along the TPB reformer's length. The dynamic region occurs up to $\pm 0.18 \mathrm{~m}$, after that the stationary state is kept until $0.60 \mathrm{~m}$. It was reported that the profiles of the endothermic reaction temperature tend to assume an inflection point at minimum values because of the endothermic reaction's effect. The range of the $\lambda_{\mathrm{s} \text {, eff. }}$ has a pertinent effect on the ERT's profiles in TPB reformer. Three different values of the $\lambda_{\mathrm{s}}$, eff. have been used to check the ERT's sensibility to the $\lambda_{\mathrm{s} \text {, eff. }}$.

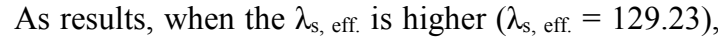
the heat absorption in TPB reformer is notably increased and therefore, the ERT's profile is favored.

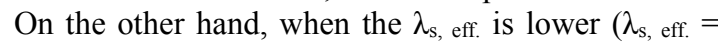
107.26), the heat absorption in TPB reformer is remarkably decreased and thus, the ERT's profile is reduced.

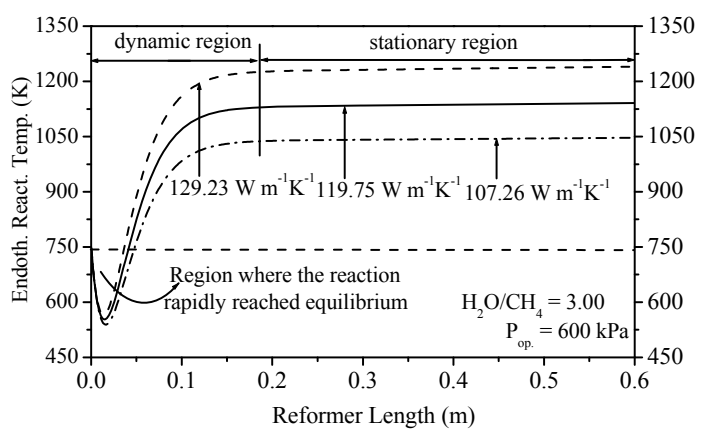

Figure 3. Endothermic reaction temperature's profiles along of the TPB reformer's length on $\beta$-SiC foam. 
Fig. 4 shows the profiles of the molar flow of each chemical component $i$ of the SRM method at the following operating conditions: $\mathrm{H}_{2} \mathrm{O} / \mathrm{CH}_{4}=3.00,500$ $\mathrm{kPa}, 1173 \mathrm{~K}$, and $1.201 \times 10^{-6}\left(\mathrm{~m}^{3} / \mathrm{s}\right)$. In this figure, the profiles of the molar flows from the consumed reactants $\left(\mathrm{H}_{2} \mathrm{O}\right.$ and $\left.\mathrm{CH}_{4}\right)$ and produced products $(\mathrm{CO}$, $\mathrm{CO}_{2}$ and $\mathrm{H}_{2}$ ) are reported in the TPB reformer. After reaching the stable state, only $18.67 \%$ of $\mathrm{H}_{2} \mathrm{O}$ was consumed. On the other hand, after achieving the stable state, $97.26 \%$ of $\mathrm{CH}_{4}$ was consumed. After reaching the stable state, $29.48 \%$ of $\mathrm{H}_{2}, 14.23 \%$ of $\mathrm{CO}_{2}$, and $2.76 \%$ of $\mathrm{CO}$ were produced for the SRM method in the TPB reformer.

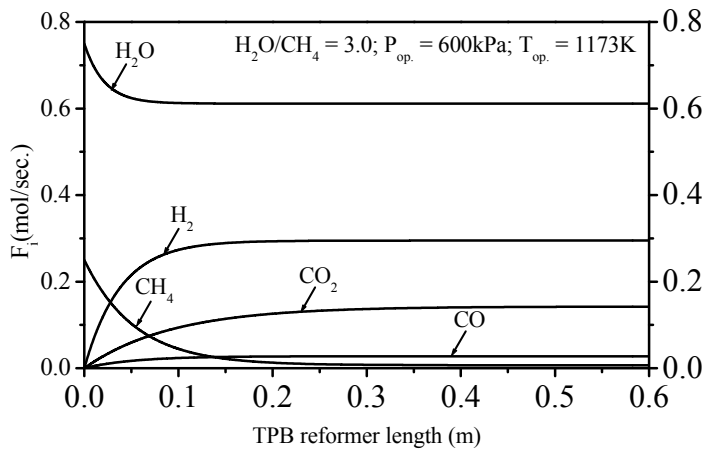

Figure 4. Molar flows of each chemical component $i$ along of the TPB reformer's length on $\beta$-SiC foam.

\section{CONCLUSIONS}

A non-isothermal pseudo-homogeneous mathematical model which includes the thermal energy transfer on the endothermic thermochemical conversion and mass transfer coupled with a thermochemical kinetic model was developed and validated against published data of the literature for a TPB reformer. After validating the numerical model, such model can be used like a process simulator. Therefore, the major conclusions are listed as follows.

1. The simulating results of molar flows are similar to experimental results of molar flows under same operating conditions. The validating results have driven a good agreement against the literature data for molar flows at the outlet from TPB.

2. When the $\lambda_{\mathrm{s}}$, eff. is notably increased, the ERT's profile is raised. On the other hand, when the $\lambda_{\mathrm{s}}$, eff. is remarkably reduced, the ERT's profile is decreased.

3. Under the prescribed operating conditions, $18.67 \%$ of $\mathrm{H}_{2} \mathrm{O}$ and $97.26 \%$ of $\mathrm{CH}_{4}$ were consumed while $29.48 \%$ of $\mathrm{H}_{2}, 14.23 \%$ of $\mathrm{CO}_{2}$, and $2.76 \%$ of $\mathrm{CO}$ were produced.

\section{ACKNOWLEDGEMENTS}

The authors of this paper would like to thank $\mathrm{CNPq}$ (National Council of Scientific and
Technological Development) for the financial support given (Process 48354/2012).

\section{REFERENCES}

Anjos E. B., Silva Filho A. M., Silva J. D., 2020, Numerical simulation of the steam reforming of toluene in a fixed-bed catalytic reformer to produce hydrogen, J Braz. Soc. Mech. Sci. Eng, Vol $42,114$.

Chen, X., Fuqiang, W., Han, Y., Yu, R., Cheng, Z., 2018, Thermochemical storage analysis of the dry reforming of methane in foam solar reactor, Energy Conversion and Management, Vol. 158, pp. 489-498.

Chompupun, T., Limtrakul, S., Vatanatham, T., Kanhari, C., Ramachandran, P. A., 2018, Experiments, modeling and scaling-up of membrane reformers for hydrogen production via steam methane reforming, Chemical Engineering Processing, Vol. 134, pp. 124-140.

Cruz, B. M., Silva, J. D., 2017, A twodimensional mathematical model for the catalytic steam reforming of methane in both conventional fixed-bed and fixed-bed membrane reformers for the production of hydrogen, International Journal of Hydrogen Energy, Vol. 42, 23670-23690.

Dias, V. F., Silva, J. D., 2020, Mathematical modelling of the solar - driven steam reforming of methanol for a solar thermochemical micro fluidized bed reformer: thermal performance and thermochemical conversion, Braz. Soc. Mech. Sci Eng., Vol. 42, pp. 447.

Fuqiang, W., Zhennan, G., Jianyu, T., Zhenyu, Y., Yu, L., 2015, Unsteady state thermochemical performance analyses of solar driven steam methane reforming in porous medium reactor, Solar Energy, Vol. 122. Pp. 1180-1192.

Hussain, A., Seidel-Morgenstern, A., Tsotsas, E., 2006, Heat and mass transfer in tubular ceramic membranes for membrane reformers, International Journal of Heat Mass Transfer, Vol. 49, pp. 22392253.

Ma, Y., He, L., Li, S., Teng, J., 2016, Heat transfer of oil shale in a small-scale fixed bed, Journal of Thermal Analysis and Calorimetry, Vol.124, pp. 461-469.

Lima, K. P. M., Dias, V. F. and Silva, J. D., 2020, Numerical modelling for the solar driven bireforming of methane for the production of syngas in a solar thermochemical micro-packed bed reformer, International Journal of Hydrogen Energy, Vol. 45, pp. 10353-10369.

Silva, J. D. and Abreu, C. A. M., 2016, Modelling and simulation in conventional fixed-bed and fixed-bed membrane reformers for the steam reforming of methane, International Journal of Hydrogen Energy, Vol. 41, pp. 11669-11674.

Vanhaecke, C., Pham-Huu, C., Edouard, D., 2012, Simulation and experimental measurement of 
dynamic behavior of solid foam filter for diesel exhaust gas, Catalysis Today, Vol. 189, pp. 101-110.

Voltolina, S., Marín, P., Díz, F. V., Salvador, O., 2017, Open-cell foams as beds in multiphase reactors: Residence time distribution and mass transfer, Chemical Engineering Journal, Vol. 316, pp. $323-331$.

Yu, T., Yuan, Q., Lu, J., Ding, J. Lu, Y., 2017, Thermochemical storage performances of methane reforming with carbon dioxide in tubular and semicavity reactors heated by a solar dish system, Applied Energy, Vol. 185, pp.1994-2004.

Appendix A: Coefficients from Eq. (10a)

$\alpha_{\mathrm{g}, 1}=\rho_{\mathrm{g}, \operatorname{mix} .} \mathrm{C}_{\mathrm{p}, \mathrm{g}, \operatorname{mix}}$.

$\alpha_{\mathrm{g}, 2}=\rho_{\mathrm{g}, \text { mix. }} C_{\mathrm{p}, \mathrm{g}, \text { mix. }} \frac{4 \mathrm{q}_{\mathrm{g}}}{\pi \mathrm{d}_{\mathrm{rz}}^{2}}$

$\alpha_{\mathrm{g}, 3}=\mathrm{h}_{\mathrm{gs}} \frac{\left(1-\varepsilon_{\mathrm{b}}\right)}{\varepsilon_{\mathrm{b}}} \frac{6}{\mathrm{~d}_{\mathrm{p}}}$

$\alpha_{\mathrm{g}, 4}=\frac{\rho_{\mathrm{g}, \text { mix. }} \mathrm{C}_{\mathrm{p}, \mathrm{g}, \text { mix. }}}{\lambda_{\mathrm{g}, \text { mix }}}$

$\alpha_{\mathrm{g}, 5}=\frac{h_{\mathrm{gs}, \text { eff. }}}{\lambda_{\mathrm{g}, \text { eff }}}$

$\lambda_{\mathrm{g}, 1}=\frac{\mathrm{L}\left(6+\mathrm{L} \alpha_{\mathrm{g}, 5}\right)\left(1+0.5 \mathrm{~L} \alpha_{\mathrm{g}, 4}\right)}{1+0.5 \mathrm{~L} \alpha_{\mathrm{g}, 5}}$

$\alpha_{\mathrm{g}, 6}=\mathrm{L}\left(6+\mathrm{L} \alpha_{\mathrm{g}, 4}\right)-\lambda_{\mathrm{g}, 1}$

$\lambda_{\mathrm{g}, 2}=\mathrm{L}^{2}\left(\alpha_{\mathrm{g}, 4} \mathrm{~T}_{\mathrm{g}, \infty}^{\mathrm{Up} .}+\alpha_{\mathrm{g}, 5} \mathrm{~T}_{\mathrm{g}, \infty}^{\text {Bot. }}\right)$

$\alpha_{g, 7}(t)=12 \bar{T}_{g}(t)+\lambda_{g, 2}\left(1+\frac{3+0.5 \mathrm{~L} \alpha_{g, 5}}{1-0.5 \mathrm{~L} \alpha_{g, 5}}\right)$

$\mathrm{T}_{\mathrm{g}}(0, \mathrm{t})=\frac{\alpha_{\mathrm{g}, 7}(\mathrm{t})}{\alpha_{\mathrm{g}, 6}}$

$\lambda_{\mathrm{g}, 3}=\frac{0.5 \mathrm{LT}_{\mathrm{g}, \infty}\left(\alpha_{\mathrm{g}, 4}+\alpha_{\mathrm{g}, 5}\right)}{1-0.5 \mathrm{~L}_{\mathrm{z}} \alpha_{\mathrm{g}, 5}}$

$\mathrm{T}_{\mathrm{g}}(\mathrm{L}, \mathrm{t})=\lambda_{\mathrm{g}, 3}-\frac{\left(1+0.5 \mathrm{~L} \alpha_{\mathrm{g}, 4}\right) \alpha_{\mathrm{g}, 7}(\mathrm{t})}{\alpha_{\mathrm{g}, 6}\left(1-0.5 \mathrm{~L}_{\mathrm{z}} \alpha_{\mathrm{g}, 5}\right)}$

Appendix B: Coefficients from Eq. (10b)

$$
\begin{aligned}
& \beta_{\mathrm{s}, 1}=\frac{\lambda_{\mathrm{s}, \text { eff. }}}{\rho_{\mathrm{s}} \mathrm{C}_{\mathrm{p}, \mathrm{s}}} \\
& \beta_{\mathrm{s}, 2}=\mathrm{h}_{\mathrm{sg}} \frac{6}{\mathrm{~d}_{\mathrm{p}}} \frac{1}{\rho_{\mathrm{s}} \mathrm{C}_{\mathrm{p}, \mathrm{s}}} \frac{\left(1-\varepsilon_{\mathrm{b}}\right)}{\varepsilon_{\mathrm{b}}}
\end{aligned}
$$

$$
\begin{aligned}
& \beta_{\mathrm{s}, 3}=\frac{\left(1-\varepsilon_{\mathrm{p}}\right)}{\varepsilon_{\mathrm{p}}} \\
& \beta_{\mathrm{s}, 4}=\frac{\mathrm{q}_{\mathrm{h}}}{\lambda_{\mathrm{s}}} \\
& \beta_{\mathrm{s}, 5}=\frac{\mathrm{h}_{\mathrm{sg}, \text { eff. }}}{\lambda_{\mathrm{s}, \text { eff. }}} \\
& \beta_{\mathrm{s}, 6}=2 \mathrm{~L}\left(3-\frac{6+\mathrm{L} \beta_{\mathrm{s}, 5}}{2+\mathrm{L} \beta_{\mathrm{s}, 5}}\right) \\
& \vartheta_{\mathrm{s}, 1}=\mathrm{L}\left(\beta_{\mathrm{s}, 4}-\beta_{\mathrm{s}, 5} \mathrm{~T}_{\mathrm{er}, \infty}\right) \\
& \vartheta_{\mathrm{s}, 2}=\frac{\mathrm{L}^{2}\left(6+\mathrm{L} \beta_{\mathrm{s}, 5}\right)\left(\beta_{\mathrm{s}, 4}+\beta_{\mathrm{s}, 5} \mathrm{~T}_{\mathrm{er}, \infty}\right)}{2+\mathrm{L} \beta_{\mathrm{s}, 5}} \\
& \beta_{\mathrm{s}, 7}(\mathrm{t})=12 \bar{T}_{\mathrm{er}}(\mathrm{t})-\vartheta_{\mathrm{s}, 1}-\vartheta_{\mathrm{s}, 2} \\
& \mathrm{~T}_{\mathrm{er}}(0, \mathrm{t})=\frac{\beta_{\mathrm{s}, 7}(\mathrm{t})}{\beta_{\mathrm{s}, 6}} \\
& \vartheta_{\mathrm{s}, 3}=\mathrm{L}\left(\beta_{\mathrm{s}, 4}+\beta_{\mathrm{s}, 5} \mathrm{~T}_{\mathrm{er}, \infty}\right)+\frac{2 \beta_{\mathrm{s}, 7}(\mathrm{t})}{2+\mathrm{L} \beta_{\mathrm{s}, 5}} \\
& \beta_{\mathrm{s}, 6}
\end{aligned}
$$

\section{Appendix C: Coefficients from Eq. (10c)}

$$
\begin{aligned}
& \varphi_{\mathrm{f}, 1}=\frac{4 \mathrm{q}_{\mathrm{g}}}{\mathrm{S}_{\mathrm{sp}} \pi \mathrm{d}_{\mathrm{ref} .}^{2} \frac{\mathrm{g}}{\mathrm{u}_{\mathrm{sg}}}} \\
& \varphi_{\mathrm{f}, 2}=\frac{\mathrm{g}}{\mathrm{u}_{\mathrm{sg}}} \frac{\mathrm{D}_{\mathrm{ax} ., \mathrm{i}}}{\mathrm{S}_{\mathrm{sp}}} \\
& \varphi_{\mathrm{f}, 3}=\frac{\mathrm{g}}{\mathrm{u}_{\mathrm{sg}}} \rho_{\mathrm{s}} \mathrm{r}_{\mathrm{ref} .}^{2} \mathrm{~L}\left(1-\varepsilon_{\mathrm{b}}\right) \\
& \varphi_{\mathrm{f}, 4}=\frac{\mathrm{L}}{\varepsilon_{\mathrm{b}}} \frac{\mathrm{u}_{\mathrm{sg}}}{\mathrm{D}_{\mathrm{ax} ., \mathrm{i}}} \\
& \varphi_{\mathrm{f}, 5}=\frac{\mathrm{L}}{\varepsilon_{\mathrm{b}}} \frac{\mathrm{k}_{\mathrm{sg}}}{\mathrm{D}_{\mathrm{ax} ., \mathrm{i}}} \\
& \mathrm{v}_{\mathrm{f}, 1}=\frac{\mathrm{L}\left(6-\mathrm{L} \varphi_{\mathrm{f}, 5}\right)}{2-\varphi_{\mathrm{f}, 5}}\left(2+\mathrm{L} \varphi_{\mathrm{f}, 4}\right) \\
& \varphi_{\mathrm{f}, 6}=\mathrm{L}_{\left(6+\mathrm{L} \varphi_{\mathrm{f}, 4}\right)-\mathrm{v}_{\mathrm{f}, 1}} \\
& \mathrm{v}_{\mathrm{f}, 2}=\mathrm{L}^{2} \mathrm{~F}_{\mathrm{i}, \infty}\left(\varphi_{\mathrm{f}, 5}-\varphi_{\mathrm{f}, 4}\right) \\
& \mathrm{v}_{\mathrm{f}, 2}=\frac{\mathrm{L}^{2} \mathrm{~F}_{\mathrm{i}, \infty}\left(6-\mathrm{L} \varphi_{\mathrm{f}, 5}\right)\left(\varphi_{\mathrm{f}, 4}+\varphi_{\mathrm{f}, 5}\right)}{2-\mathrm{L}_{\mathrm{f}, 5}}
\end{aligned}
$$




$$
\begin{gathered}
\varphi_{\mathrm{f}, 7}(\mathrm{t})=12 \overline{\mathrm{F}}_{\mathrm{i}}(\mathrm{t})-\mathrm{v}_{\mathrm{f}, 2}-\mathrm{v}_{\mathrm{f}, 3} \\
\mathrm{~F}_{\mathrm{i}}(0, \mathrm{t})=\frac{\varphi_{\mathrm{f}, 7}(\mathrm{t})}{\varphi_{\mathrm{f}, 6}} \\
\mathrm{v}_{\mathrm{f}, 4}=\left(2+\mathrm{L} \varphi_{\mathrm{f}, 4}\right) \frac{\varphi_{\mathrm{f}, 7}(\mathrm{t})}{\varphi_{\mathrm{f}, 6}} \\
v_{\mathrm{f}, 5}=\mathrm{LF}_{\mathrm{i}, \infty}\left(\varphi_{\mathrm{f}, 4}+\varphi_{\mathrm{f}, 5}\right) \\
\mathrm{F}_{\mathrm{i}}(\mathrm{L}, \mathrm{t})=\frac{\left(\mathrm{v}_{\mathrm{f}, 4}-\mathrm{v}_{\mathrm{f}, 5}\right)}{2-\mathrm{L} \varphi_{\mathrm{f}, 5}}
\end{gathered}
$$

